\title{
Independent and Leader-Follower Control for Two Magnetic Micro-agents
}

\author{
Alper Denasi and Sarthak Misra
}

\begin{abstract}
Microrobotics is a promising field that can revolutionize fields such as minimally invasive surgery. Applications such as micro-manipulation can be performed more effectively using multiple micro-sized agents. These can be performed more accurately with the help of robust controllers. In this paper, we design a leader-follower controller that can be used to perform coordinated motion tasks. A prescribed performance controller is designed for the leader micro-agent whereas a synchronization controller is designed for the follower. The main difference between our method and the literature is that our method can achieve a pre-specified control performance. The position of the micro-agents are obtained using microscopic images and image processing. The velocities and accelerations of the microagents are obtained using state observers. The algorithm is tested experimentally on spherical magnetic microparticles that have an average diameter of $100 \mu \mathrm{m}$. Two types of experiments are performed. The first one is related to the leader-follower control, whereas the second one demonstrates the independent control of the two agents. The maximum value of the steady-state errors obtained in the leader-follower control experiments are $14.45 \mu \mathrm{m}$ and $10.19 \mu \mathrm{m}$ in $x$ - and $y$ - directions for the leader agent and 6.47 $\mu \mathrm{m}$ and $7.77 \mu \mathrm{m}$ in $\mathrm{x}$ - and $\mathbf{y}$-directions for the follower errors, respectively.
\end{abstract}

Index Terms-Micro/nano robots, automation at micronano scales, motion control.

\section{INTRODUCTION}

$\mathbf{U}$ TILIZING teams of micro- and/or nanorobots instead of individual ones can facilitate their application to minimally invasive surgery, assembly and environmental remediation. The completion time of tasks can be reduced with the help of multiple robots. That being said, cooperative control of multiple microrobots has its own challenges [1]. It is difficult to embed onboard sensors and actuators on microrobots. Thus, such micro-agents are often designed to be magnetic and actuated wirelessly. In the case of magnetic actuation using a set of coils placed around the workspace, all the micro-agents receive the same control inputs (currents to be more specific). Consequently, the number of controllable degrees-of-freedom of the multiple micro-agent system is directly related to the number coils of the experimental setup [2].

Research on the control of multiple micro-agents has been done both considering their independent and cooperative con-

Manuscript received: March, 14, 2017; Revised June, 14, 2017; Accepted July, 17, 2017.

This paper was recommended for publication by Editor Yu Sun upon evaluation of the Associate Editor and Reviewers' comments. This project (ROBOTAR) has received funding from the European Research Council (ERC) under the European Union's Horizon 2020 Research and Innovation programme (Grant Agreement \#638428).

A. Denasi and S. Misra are affiliated with the Surgical Robotics Laboratory, Department of Biomechanical Engineering, MIRA-Institute for Biomedical Technology and Technical Medicine, University of Twente, The Netherlands. S. Misra is also affiliated with the Department of Biomedical Engineering, University of Groningen and University Medical Centre Groningen, The Netherlands. a.denasidutwente.nl, s.misra@utwente.nl

Digital Object Identifier (DOI): see top of this page.

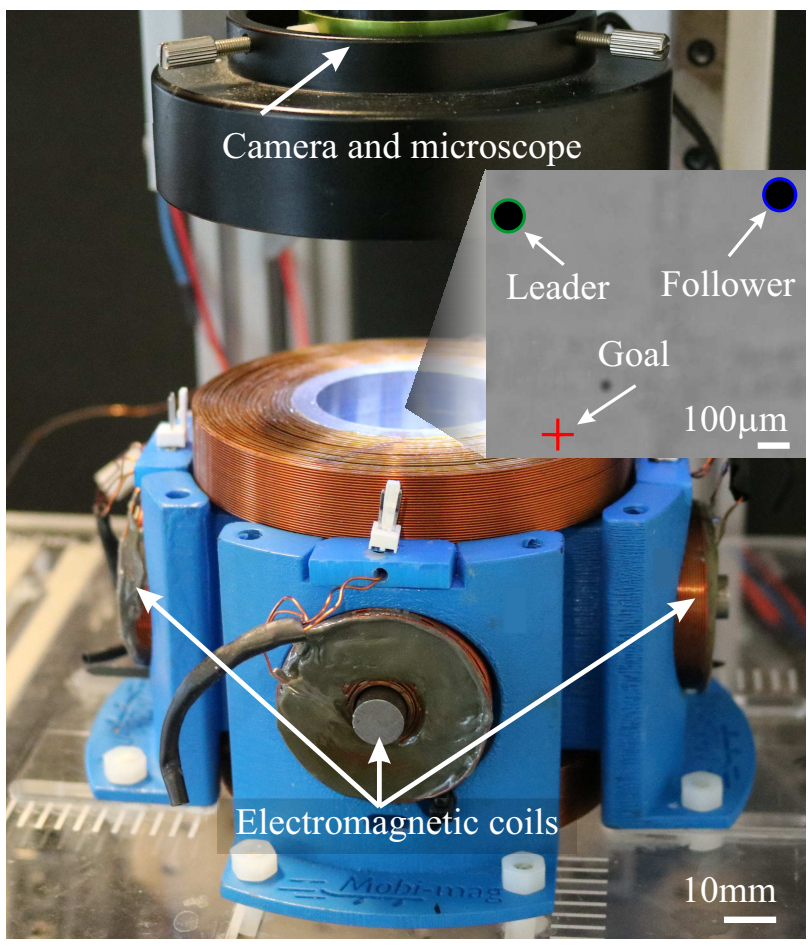

Fig. 1. The Mobi-Mag electromagnetic system used to control multiple magnetic micro-agents using camera feedback. The positions of the microagents are obtained using an image processing algorithm. In order to enable the cooperation of multiple micro-agents, a leader-follower controller is developed that uses the aforementioned feedback. An optimization algorithm computes the control currents which are sent to the DC servo drives which drive the electromagnetic coils.

trol. For instance, the independent control of two millimeterscale permanent magnets was investigated by using PID controllers and a Levenberg-Marquardt solver [3]. Another approach was based on using a combination of rotating fields and proportional and lag-angle feedback controllers upto three magnetic micro-agents in three dimensions [4]. The Levenberg-Marquardt solver requires the precomputation of the currents and lag-angle feedback controller requires a priori calibration which are both dependent on the precise knowledge of the dynamic model of the agents and external disturbances. Cooperative open-loop control of multiple magnetic microagents is investigated using a conical permanent magnet attached to a robotic manipulator to steer the microrobots [5]. Instead of designing a controller for each agent in cooperative control, the mean position of the swarm together with its variance are controlled in a simulated block-pushing task [2]. The application of the method to magnetic micro-agents is not straightforward since the agent dynamics is nonlinear with respect to the control inputs. Chao et al. presented a twolevel planning framework in which a steering direction was computed for a swarm of magnetic nanoparticles in an openloop manner [6]. The influence of environmental disturbances 
such as fluid flow and navigation in a microfluidic channel using an optimal controller has been studied for two magnetic microbeads [7]. In [7], though the results were promising, it was difficult to judge the asymptotic stability of the controller from the experiments. Besides the studies on the control of multiple magnetic beads, the control of multiple magnetotactic bacteria has also been investigated starting with the work of Martel and Mohammadi [8]. Multiple artificially magnetotactic bacteria are steered using ensemble control which has initially been applied to the control of multiple unicycle type mobile robots [1], [9], [10]. Chowdhury et al. presented a survey on the state-of-the art for controlling multiple microrobots [11].

Multiple microrobots can perform coordinated and cooperative tasks with the help of controlled synchronization [12]. The automation of these tasks require using different modes of control, including the independent control of the agents at the initial phase of the task. A basic example of coordinated motion tasks is leader-follower type of task. In this work, we designed a leader-follower controller for two paramagnetic micro-sized agents. We obtain estimates of the positions of the micro-agents with respect to an inertial frame using an image processing algorithm. These positions are utilized to estimate the velocity and acceleration of the agents using suitable state observers. These signals are used in the design of a prescribed performance controller for the leader and a passivity-based synchronization controller for the follower micro-agent. The control forces are converted to currents using a relaxed semidefinite optimization approach. We designed a prescribed performance controller for an independent control task. Our approach differs from previous work in that the currents are also minimized to reduce the electrical power. The optimization algorithm is fast and does not require a robust initial solution contrary to Newton-based methods. Further, precomputation of the control currents or a priori calibration procedures are not required for our approach.

The major contributions of this work are:

- A leader-follower controller and an independent controller for micro-agents that can cope with modeling uncertainties and disturbances (Section III-A),

- A fast and initialization-free optimization-based solver that can minimize the electrical power (Section III-B),

- Experimental demonstration of pre-specified control performance of both algorithms using two paramagnetic microparticles on different trajectory following tasks (Fig. 1) (Section IV).

\section{Modeling Magnetic Micro-Agents}

In this section, we provide a mathematical model for microagents whose primary mode of actuation is electromagnetic. Further, the micro-agents move in a fluidic environment and contain soft magnetic material. Let $\{I\}$ be inertial frame and $\{B\}$ be a body frame fixed to the center of mass of the micro-agent, respectively. The position vector $\mathbf{p}_{B / I}^{I}(t) \in \mathbb{R}^{3}$, from $\{I\}$ to $\{B\}$ expressed in $\{I\}$ is given by $\mathbf{p}_{B / I}^{I}(t)=$ $\left[\begin{array}{lll}x(t) & y(t) & z(t)\end{array}\right]^{T}$. Here, $t \in \mathbb{R}$ represents the time. The translational equations of motion of the magnetic micro-agent are given as follows:

$$
M_{p} \ddot{\mathbf{p}}_{B / I}^{I}(t)=\mathbf{F}_{d}\left(\dot{\mathbf{p}}_{B / I}^{I}(t)\right)+\mathbf{F}_{m a g}\left(\mathbf{p}_{B / I}^{I}(t)\right)+\mathbf{F}_{b}+\mathbf{F}_{g}
$$

where $M_{p} \in \mathbb{R}_{>0}$ is the mass of the micro-agent. Further, $\mathbf{F}_{d}\left(\dot{\mathbf{p}}_{B / I}^{I}(t)\right) \in \mathbb{R}^{3}, \mathbf{F}_{\text {mag }}\left(\mathbf{p}_{B / I}^{I}(t)\right) \in \mathbb{R}^{3}, \mathbf{F}_{b} \in \mathbb{R}^{3}$ and $\mathbf{F}_{g} \in \mathbb{R}^{3}$ are the hydrodynamic drag force, the magnetic force, buoyancy force and the weight, respectively. We assume that the micro-agents operate in the low Reynolds hydrodynamic regime. For a spherically shaped micro-agent, according to the Stokes' law the drag force can be computed as follows:

$$
\mathbf{F}_{d}\left(\dot{\mathbf{p}}_{B / I}^{I}(t)\right)=-6 \pi \eta_{f} r_{p} \dot{\mathbf{p}}_{B / I}^{I}(t),
$$

where $\eta_{f}$ and $r_{p}$ are the dynamic viscosity of the fluid and the radius, respectively [13]. The weight and buoyancy forces are given as follows:

$$
\mathbf{F}_{g}=V_{p} \rho_{p} \mathbf{g}, \quad \mathbf{F}_{b}=-V_{p} \rho_{f} \mathbf{g},
$$

where $V_{p}$ and $\mathbf{g}$ are the volume of the micro-agent and gravitational acceleration and $\rho_{p}, \rho_{f}$ are the density of the microagent and fluid, respectively. The influence of the capillary forces can also be included in the dynamics formulation [14]. However, in our case the reservoir in which the micro-agents are placed is sufficiently large so that the capillary effect can be neglected (see Section IV). Finally, the magnetic forces exerted by an array of electromagnets on the micro-agent should be included to the model. Let $n$ be the number of electromagnetic coils. We assume that the magnetic field of each coil varies linearly in the workspace where the microagents are controlled. Thus, the total magnetic flux density is computed by the superposition of the contribution of the $i^{\text {th }}$ coil as follows:

$$
\mathbf{B}\left(\mathbf{p}_{B / I}^{I}\right)=\sum_{i=1}^{n} \widetilde{\mathbf{B}}_{i}\left(\mathbf{p}_{B / I}^{I}\right) \mathcal{I}_{i}=\widetilde{\mathbf{B}}\left(\mathbf{p}_{B / I}^{I}\right) \mathcal{I}
$$

where $\widetilde{\mathbf{B}}\left(\mathbf{p}_{B / I}^{I}\right) \in \mathbb{R}^{3 \times n}$ is a position-dependent matrix evaluated at $\mathbf{p}_{B / I}^{I}$ and $\mathcal{I} \in \mathbb{R}^{n \times 1}$ is the vector of applied currents [15]. The magnetic force $\mathbf{F}_{m a g} \in \mathbb{R}^{3}$ that the micro-agent experiences acting at a point $\mathbf{p}_{B / I}^{I}$ is given by

$$
\mathbf{F}_{\text {mag }}\left(\mathbf{p}_{B / I}^{I}\right)=\nabla\left(\mathbf{m} \cdot \mathbf{B}\left(\mathbf{p}_{B / I}^{I}\right)\right),
$$

where $\mathbf{m} \in \mathbb{R}^{3}$ and $\mathbf{B}\left(\mathbf{p}_{B / I}^{I}\right) \in \mathbb{R}^{3}$ are the magnetic dipole moment and the global magnetic field given by (4), respectively. For soft magnetic materials with a spherical shape, neglecting hysteresis and saturation, the magnetic dipole moment is computed as follows:

$$
\mathbf{m}\left(\mathbf{p}_{B / I}^{I}\right)=k_{m a g} \mathbf{B}\left(\mathbf{p}_{B / I}^{I}\right)=\frac{\chi_{m} V_{p}}{\mu_{0}\left(1+\chi_{m}\right)} \mathbf{B}\left(\mathbf{p}_{B / I}^{I}\right),
$$

where $\chi_{m}$ and $\mu_{0}$ are the magnetic susceptibility and the vacuum permeability, respectively [16]. Here, $k_{\text {mag }}$ is introduced to combine the magnetic parameters into a single constant. Consequently, the forces are related to the currents via the following map:

$$
\mathbf{F}_{m a g}\left(\mathbf{p}_{B / I}^{I}, \mathcal{I}\right)=k_{m a g}\left[\begin{array}{l}
\mathcal{I}^{T} \frac{\partial}{\partial p_{B / I}^{I, x}}\left(\widetilde{\mathbf{B}}^{T}\left(\mathbf{p}_{B / I}^{I}\right) \widetilde{\mathbf{B}}\left(\mathbf{p}_{B / I}^{I}\right)\right) \mathcal{I} \\
\mathcal{I}^{T} \frac{\partial}{\partial p_{B / I}^{I, y}}\left(\widetilde{\mathbf{B}}^{T}\left(\mathbf{p}_{B / I}^{I}\right) \widetilde{\mathbf{B}}\left(\mathbf{p}_{B / I}^{I}\right)\right) \mathcal{I} \\
\mathcal{I}^{T} \frac{\partial}{\partial p_{B / I}^{I, z}}\left(\widetilde{\mathbf{B}}^{T}\left(\mathbf{p}_{B / I}^{I}\right) \widetilde{\mathbf{B}}\left(\mathbf{p}_{B / I}^{I}\right)\right) \mathcal{I}
\end{array}\right] .
$$


The currents corresponding to the desired magnetic forces are obtained by solving the inverse of the quadratic relations in (7) using the optimization technique described in Section III-B.

\section{Controller Design}

In this section, we describe the leader-follower controller to enable the cooperation of two micro-agents. The control scheme can be extended to control more micro-agents which requires more magnetic coils. Consequently, the minimum required number of coils is the product of number of agents and number of degrees of freedom needed to control. In what follows, we omit the subscripts and superscripts regarding the frames of the position and velocity variables. Instead, we introduce subscripts for the leader $(l)$ and follower $(f)$ microagents, also known as the master and slave, respectively. First, the formation controller is introduced. This is followed by the formulation of the optimization algorithm.

\section{A. Leader-Follower based Formation Controller}

The leader-follower approach ensures the cooperation of multiple micro-agents where the leader is driven by a desired trajectory and the follower tracks the leader with a suitable synchronization controller. The controllers required for both agents make use of position estimates obtained from the image processing algorithm [17]. The pseudocode of the image processing algorithm is presented in Algorithm 1. The velocity and acceleration information for both controllers is obtained with the help of state observers. We first design a trajectory tracking controller for the leader based on the prescribed performance approach described in [18], [19]. For this purpose, let us first define the position and velocity tracking errors for the leader as follows:

$$
\begin{aligned}
\mathbf{e}_{l} & =\mathbf{p}_{l}-\mathbf{p}_{r}, \\
\dot{\mathbf{e}}_{l} & =\dot{\mathbf{p}}_{l}-\dot{\mathbf{p}}_{r},
\end{aligned}
$$

where $\mathbf{e}_{l} \in \mathbb{R}^{3}$ and $\dot{\mathbf{e}}_{l} \in \mathbb{R}^{3}$. Since the velocity $\dot{\mathbf{p}}_{l} \in \mathbb{R}^{3}$ is not directly measured, its estimate $\widehat{\dot{\mathbf{p}}}_{l}$ obtained from the iterative learning observer is used [19]. The reference positions, velocities and accelerations are $\mathbf{p}_{r} \in \mathbb{R}^{3}, \dot{\mathbf{p}}_{r} \in \mathbb{R}^{3}$ and $\ddot{\mathbf{p}}_{r} \in \mathbb{R}^{3}$, respectively. The combined position and velocity error can be defined as follows:

$$
\mathbf{s}_{l}=\dot{\mathbf{e}}_{l}+\mathbf{\Lambda}_{l} \mathbf{e}_{l},
$$

where $\Lambda_{l} \in \mathbb{R}^{3 \times 3}$ is a diagonal positive definite gain matrix. The combined error described by (10) draws its origins from the sliding mode theory. It is a stable linear filter with the input $\mathbf{s}_{l} \in \mathbb{R}^{3}$ and output $\mathbf{e}_{l}$, respectively. According to the prescribed performance strategy, using the combined error (10), the following controller $\mathbf{F}_{l, i}$ is selected for the leader agent:

$$
\mathbf{F}_{l, i}=-k_{l, i} \ln \left(\frac{1+\frac{s_{l, i}}{\rho_{l, i}}}{1-\frac{s_{l, i}}{\rho_{l, i}}}\right) \quad \text { for } \quad i \in\{x, y, z\},
$$

where $k_{l, i}>0$ is the control gain. In (11), the performance function $\rho_{l, i} \in \mathbb{R}$ on the combined position and velocity error (10) is defined as follows:
Algorithm 1 The details of the image processing algorithm for tracking a spherical micro-agent are presented. At the beginning of the closed-loop control experiment, each microagent is identified with a user interface. Further, a fixed-size rectangular region of interest (ROI) is formed around each micro-agent with its center matching the center coordinates of the micro-agent. $\mathbf{I}_{R G B}$ represents the image in RGB format.

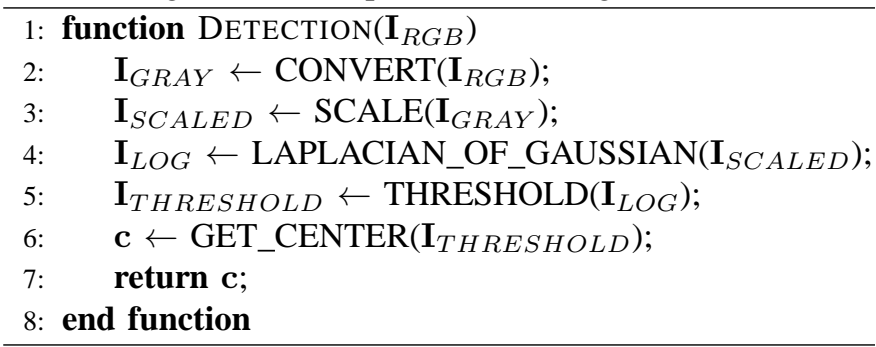

$$
\rho_{l, i}=\left(\rho_{l, i, 0}-\rho_{l, i, \infty}\right) e^{-\alpha_{l, i} t}+\rho_{l, i, \infty},
$$

where $\rho_{l, i, 0}, \rho_{l, i, \infty}$ and $\alpha_{l, i}$ are the initial and final bounds on the combined error and the decay rate, respectively. The natural logarithm function given in (11) represents a coordinate transformation as a function of the scaled error $s_{l, i} / \rho_{l, i}$. When the initial value of the combined error (10) satisfies $\left|s_{l, i}(0)\right|<$ $\rho_{l, i}(0)$, the controller (11) guarantees the boundedness of $s_{l, i}(t)$ for $t \rightarrow \infty$ [20]. In order for the second agent to follow the leader, a trajectory tracking controller is designed using the passivity-based control technique. In passivity-based control, the nonlinearities in the system dynamics (such as drag forces) are compensated using reference trajectories instead of exact cancellation. Considering the uncertainties in microagent dynamics, the controller for the follower is selected as follows:

$\mathbf{F}_{f}=\widehat{M}_{f} \widehat{\ddot{\mathbf{p}}}_{l}+\widehat{\mathbf{F}}_{d, f}\left(\widehat{\dot{\mathbf{p}}}_{l}\right)-\widehat{\mathbf{F}}_{b, f}-\widehat{\mathbf{F}}_{g, f}-\mathbf{K}_{d, l f} \widehat{\dot{\mathbf{e}}}_{l f}-\mathbf{K}_{p, l f} \mathbf{e}_{l f}$,

where $\widehat{\mathbf{F}}_{d, l}, \widehat{\mathbf{F}}_{b, l}, \widehat{\mathbf{F}}_{g, l} \in \mathbb{R}^{3}$ and $\widehat{M}_{f} \in \mathbb{R}_{>0}$ are the estimates of the drag, buoyancy and gravity forces and the mass, respectively. $\mathbf{K}_{p, l f} \in \mathbb{R}^{3 \times 3}$ and $\mathbf{K}_{d, l f} \in \mathbb{R}^{3 \times 3}$ are positive definite gain matrices for the synchronization error $\mathbf{e}_{l f} \in \mathbb{R}^{3}$ and its estimated time derivative $\widehat{\dot{\mathbf{e}}}_{l f} \in \mathbb{R}^{3}$, respectively. The synchronization error in (13) is defined as follows:

$$
\mathbf{e}_{l f}=\mathbf{p}_{f}-\mathbf{p}_{l}-\mathbf{d}_{l f},
$$

where $\mathbf{d}_{l f} \in \mathbb{R}^{3}$ determines the formation distance between the leader and follower micro-agents. The vector $\mathbf{d}_{l f}$ could be selected to be time-varying to obtain different formation behaviors. For the sake of simplicity we assume it to be constant in this work. Further, the variables $\widehat{\dot{\mathbf{p}}}_{l}$ and $\widehat{\ddot{\mathbf{p}}}_{l}$ are estimates for the velocity and acceleration of the leader microagent, respectively. These are obtained by using two separate state observers; one for the synchronization error and the other for the follower variables [12]. The following state observer is designed to estimate the synchronization error and its time derivative:

$$
\begin{aligned}
\frac{d}{d t} \widehat{\mathbf{e}}_{l f} & =\widehat{\dot{\mathbf{e}}}_{l f}+\mathbf{L}_{l f, 1} \widetilde{\mathbf{e}}_{l f}, \\
\frac{d}{d t} \widehat{\dot{\mathbf{e}}}_{l f} & =-\widehat{M}_{f}^{-1}\left(\mathbf{K}_{d, l f} \widehat{\dot{\mathbf{e}}}_{l f}+\mathbf{K}_{p, l f} \widehat{\mathbf{e}}_{l f}\right)+\mathbf{L}_{l f, 2} \widetilde{\mathbf{e}}_{l f},
\end{aligned}
$$


where $\mathbf{L}_{l f, 1} \in \mathbb{R}^{3 \times 3}$ and $\mathbf{L}_{l f, 2} \in \mathbb{R}^{3 \times 3}$ are positive definite gain matrices. The estimation error $\widetilde{\mathbf{e}}_{l f}$ for the synchronization error is defined as follows:

$$
\widetilde{\mathbf{e}}_{l f}=\mathbf{e}_{l f}-\widehat{\mathbf{e}}_{l f},
$$

where $\widehat{\mathbf{e}}_{l f}$ is an estimate of $\mathbf{e}_{l f}$. The following state observer is designed to estimate the follower micro-agent position and velocity

$$
\begin{aligned}
\frac{d}{d t} \widehat{\mathbf{p}}_{f} & =\widehat{\dot{\mathbf{p}}}_{f}+\mathbf{L}_{f, 1} \widetilde{\mathbf{p}}_{f}, \\
\frac{d}{d t} \widehat{\mathbf{p}}_{f} & =-\widehat{M}_{f}^{-1}\left(\mathbf{K}_{d, l f} \widehat{\dot{\mathbf{e}}}_{l f}+\mathbf{K}_{p, l f} \mathbf{e}_{l f}\right)+\mathbf{L}_{f, 2} \widetilde{\mathbf{p}}_{f},
\end{aligned}
$$

where $\mathbf{L}_{f, 1} \in \mathbb{R}^{3 \times 3}$ and $\mathbf{L}_{f, 2} \in \mathbb{R}^{3 \times 3}$ are positive definite gain matrices. In equations (18) and (19), the estimation error for the follower position is defined as follows:

$$
\widetilde{\mathbf{p}}_{f}=\mathbf{p}_{f}-\widehat{\mathbf{p}}_{f},
$$

where $\widehat{\mathbf{p}}_{f}$ is an estimate of $\mathbf{p}_{f}$. The estimates of the leader velocity $\hat{\mathbf{p}}_{l}$ and acceleration $\widehat{\ddot{\mathbf{p}}}_{l}$ used in the follower controller (13) can be obtained using (16) and (19). The estimated leader velocity $\widehat{\dot{\mathbf{p}}}_{l}$ can be obtained using the definition of the synchronization error (14) as follows:

$$
\widehat{\dot{\mathbf{p}}}_{l}=\widehat{\dot{\mathbf{p}}}_{f}-\widehat{\dot{\mathbf{e}}}_{l f},
$$

where it was assumed that $\dot{\mathbf{d}}_{l f}=0$. The estimated leader acceleration $\widehat{\ddot{\mathbf{p}}}_{l}$ can be obtained using (16) and (19) the following algebraic equation:

$\widehat{\ddot{\mathbf{p}}}_{l}=\frac{d}{d t}\left(\widehat{\dot{\mathbf{p}}}_{f}-\widehat{\dot{\mathbf{e}}}_{l f}\right)=-\left(\widehat{M}_{f}^{-1} \mathbf{K}_{p, l f}+\mathbf{L}_{p, l f}\right) \widetilde{\mathbf{e}}_{l f}+\mathbf{L}_{p, l f} \widetilde{\mathbf{p}}_{f}$.

\section{B. Optimization Routine to Obtain Control Currents}

In this section, we describe an optimization based solution to obtain the control currents corresponding to the magnetic forces obtained using the aforementioned leader (11) and formation (13) controllers. Instead of just solving for the currents using the relation (7) we also minimize the electrical power of the system by introducing a suitable cost function. Consequently, the optimization problem to be solved can be formulated as follows:

$$
\begin{array}{rl}
\min _{\mathcal{I} \in \mathbb{R}^{n}} & \mathcal{J}(\mathcal{I}) \\
\text { s.t. } & \mathbf{h}_{e q}(\mathcal{I})=\mathbf{0},
\end{array}
$$

where $\mathcal{J}(\mathcal{I}) \in \mathbb{R}$ and $\mathbf{h}_{e q}(\mathcal{I}) \in \mathbb{R}^{6}$ are the objective and equality constraint functions, respectively [21]. The objective function is quadratic and is selected as follows:

$$
\mathcal{J}(\mathcal{I})=\frac{1}{2} \mathcal{I}^{T} \mathcal{I}
$$

The relation between the magnetic control forces and the coil currents for the leader and follower can be introduced as equality constraints $\mathbf{h}_{e q}(\mathcal{I})$ as follows:

$$
\mathbf{h}_{e q}(\mathcal{I})=\left[\begin{array}{c}
\mathbf{F}_{\text {mag }}\left(\mathbf{p}_{l}, \mathcal{I}\right)-\mathbf{F}_{l} \\
\mathbf{F}_{\text {mag }}\left(\mathbf{p}_{f}, \mathcal{I}\right)-\mathbf{F}_{f}
\end{array}\right],
$$

where $\mathbf{F}_{l}$ and $\mathbf{F}_{f}$ are computed using the controller expressions (11) and (13), respectively. For the considered functions, this problem falls under the class of quadratically constrained quadratic programs (QCQPs) [22]. It can be reformulated as a non-convex semi-definite optimization problem as follows:

$$
\begin{array}{ll}
\min _{\mathfrak{I} \in \mathbb{S}^{n}} \operatorname{tr}(\mathfrak{I}) \\
\text { s.t. } \operatorname{tr}\left[k_{\text {mag }}\left(\frac{\partial}{\partial p_{l, i}}\left(\widetilde{\mathbf{B}}^{T}\left(\mathbf{p}_{l}\right) \widetilde{\mathbf{B}}\left(\mathbf{p}_{l}\right)\right)\right) \mathfrak{I}\right]-F_{l, i}=0 \\
\operatorname{tr}\left[k_{\text {mag }}\left(\frac{\partial}{\partial p_{f, i}}\left(\widetilde{\mathbf{B}}^{T}\left(\mathbf{p}_{f}\right) \widetilde{\mathbf{B}}\left(\mathbf{p}_{f}\right)\right)\right) \mathfrak{I}\right]-F_{f, i}=0 \\
\mathfrak{I} \geq 0, \quad \operatorname{rank}(\mathfrak{I})=1
\end{array}
$$

for $i \in\{x, y, z\}$ where $\mathbb{S}^{n}$ represents the set of all $n \times n$ real-symmetric matrices and $\mathfrak{I}=\mathcal{I I}^{T} \geq 0$ indicates that the matrix $\mathfrak{I}$ is positive semi definite. Further, $\operatorname{tr}()$ indicates the matrix trace operation. Since the rank equality constraint is not a convex constraint, it can be dropped and instead the relaxed semi-definite optimization problem can be solved to obtain the globally optimal solution $\mathfrak{I}^{*}$. The main issue with this technique is how to extract the optimal currents $\mathcal{I}^{*}$ from this solution, since the rank of $\mathfrak{I}^{*}$ can be larger than 1 . One possible way to obtain the optimal currents is to compute the rank-one approximation of $\mathfrak{I}^{*}$. Specifically, let $r=\operatorname{rank}(\mathfrak{I})<$ $n$, and let

$$
\mathfrak{I}^{*}=\sum_{i=1}^{r} \lambda_{i} \mathbf{v}_{i} \mathbf{v}_{i}^{T},
$$

indicate the eigen-decomposition of $\mathfrak{I}$, where $\lambda_{1} \geq \lambda_{2} \geq$ $\ldots \lambda_{r}>0$ are the eigenvalues and $\mathbf{v}_{1}, \ldots, \mathbf{v}_{r} \in \mathbb{R}^{n}$ are the respective eigenvectors. The best rank-one approximation $\mathfrak{I}_{1}^{*}$ to $\mathfrak{I}^{*}$ (in the least two-norm sense) is given by $\mathfrak{I}_{1}^{*}=\lambda_{1} \mathbf{v}_{1} \mathbf{v}_{1}^{T}$. Thus, the optimal current is obtained as follows:

$$
\mathcal{I}^{*}=\sqrt{\lambda_{1}} \mathbf{v}_{1}
$$

The block diagram of the control system is shown in Fig 2.

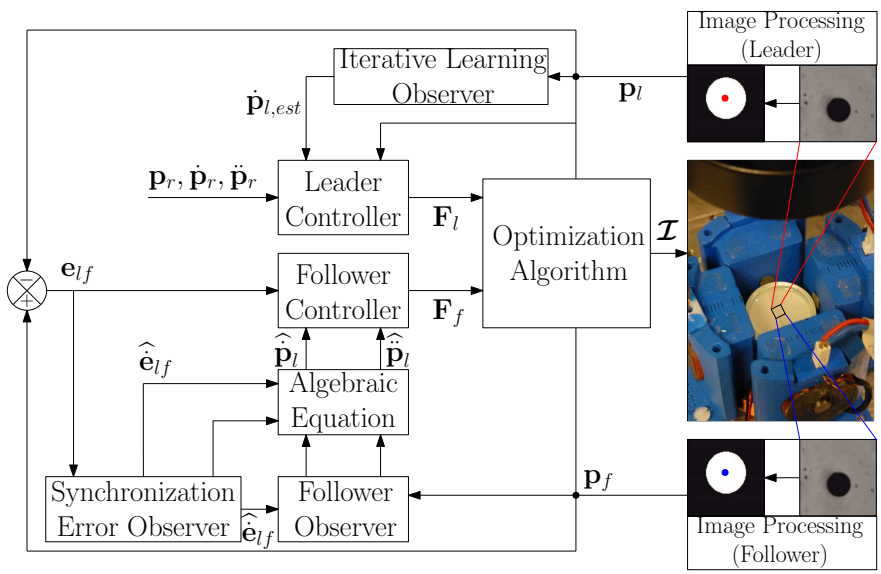

Fig. 2. The block diagram of leader-follower controller is presented. The controller for leader and follower are given by (11) and (13), respectively. The observer for follower variables and synchronization error are given by (19) and (16), respectively. Further, the algebraic equation is given by (22). Finally, the optimization algorithm is given by (26).

\section{EXPERIMENTS}

In this section, we start by briefly introducing our experimental setup followed by the results related to the formation 
TABLE I

CONTROLLER AND OBSERVER PARAMETERS

\begin{tabular}{cccc}
\hline Parameter & Leader & Parameter & Follower \\
\hline $\boldsymbol{\Lambda}_{l}$ & $\operatorname{diag}[4,4]$ & $\mathbf{K}_{p, l f}$ & $\operatorname{diag}[150,150]$ \\
\hline$k_{l, x}, k_{l, y}$ & 750 & $\mathbf{K}_{d, l f}$ & $\operatorname{diag}[20,20]$ \\
\hline$\rho_{l, x, 0}, \rho_{l, y, 0}$ & 10 & $\mathbf{L}_{l f, 1}$ & $\operatorname{diag}[50,50]$ \\
\hline$\rho_{l, x, \infty}, \rho_{l, y, \infty}$ & 2.5 & $\mathbf{L}_{l f, 2}$ & $\operatorname{diag}[2500,2500]$ \\
\hline$\alpha_{l, x}, \alpha_{l, y}$ & 0.5 & $\mathbf{L}_{f, 1}$ & $\operatorname{diag}[20,20]$ \\
\hline & & $\mathbf{L}_{f, 2}$ & $\operatorname{diag}[400,400]$ \\
\hline
\end{tabular}

controller. Finally, experiments regarding the independent control of two microparticles are presented.

\section{A. Experimental Setup}

The setup consists of an array of 6 electromagnetic coils with iron cores placed orthogonally around a fluid reservoir (Fig. 1). We used four of these coils that lie on the same plane to enable the planar manipulation of the micro-agents. Further details of the individual components of the setup can be found in our prior work [19]. The assumption about the linearity of the total magnetic field density as a function of the coil currents mentioned in Section II is valid upto 1.9 [A] for each of the coils in the setup. The iron cores saturate above this current value. We used paramagnetic microparticles with an average diameter of $100 \mu \mathrm{m}$, consisting of ironoxide in a poly(lactic acid) matrix (PLA Particles-M-redFplain from Micromod Partikeltechnologie $\mathrm{GmbH}$, RostockWarnemuende, Germany). The specific value of the diameters of the microparticles are $134 \mu \mathrm{m}$ and $144 \mu \mathrm{m}$, respectively. The parameters regarding their magnetic properties including other physical parameters can be found in our previous work [19]. We conducted all experiments in a commercial Petri dish with a diameter of 40 millimeters and a height of 12 millimeters. For such a reservoir size, the influence of the capillary forces is considered to be negligible. Both of the leader-follower and independent control experiments are conducted in water at room temperature. Mosek version 7.0 (Mosek ApS, Denmark) is used to implement interior point based optimization routine to solve the relaxed version of the semi-definite programming problem defined by (26) in Section III-B. Eigen C++ library is used for linear algebraic operations such as the eigen-decomposition mentioned in Section III-B. The sampling frequency is set at $25 \mathrm{~Hz}$.

\section{B. Leader-Follower Control Results}

We present the representative results of the leader-follower type formation control experiments for the algorithm introduced in Section III. In these experiments, the A quintic polynomial reference trajectory with zero initial and final velocities and accelerations is used to drive the leader microagent. The end-point of the reference trajectory is obtained by clicking with the mouse on the graphical user interface. The final time of the reference trajectory is selected as $t_{f}=15$ seconds. The controllers and observers gains for the leader and follower agents are provided in Table I. These values are determined empirically considering the actuator limits such as the maximum current and the cut-off frequency of the coils.

Using these tuning parameters, the combined position and velocity error (10) $s_{l, i}(t)$ for $\mathrm{x}$ - and $\mathrm{y}$ - directions shown in Fig. 3 are obtained. Further, the exponentially decaying performance functions $\rho_{l, i}(t)$ are also shown on each plot in Fig. 3. In all cases, the combined error remained within the prescribed performance bounds. In order to quantitatively evaluate the performance of the controllers, Maximum Absolute Error (M.A.E.), Integral of Absolute Error (I.A.E.), Integral of Squared Error (I.S.E.) for the leader and follower agents are computed which are presented in Table II. Video snapshots of the leader and follower agents during the formation control experiment are shown in Fig. 4.

The controller forces for the leader and follower agents given by (11) and (13) and the forces obtained with (7) using the optimal current (28) for $\mathrm{x}$ - and $\mathrm{y}$ - directions are shown in Fig. 5. The quantitative evaluation of the performance of the optimization algorithm for the leader and follower forces are presented in Table III. It can be realized from Fig. 5 that there are time instances where the force errors are relatively high however the controllers for the leader (11) and follower (13) are robust enough to cope with this. The optimal currents obtained from (28) after the relaxed semi definite optimization problem (26) is solved are between -1 and 1 Amperes.

TABLE II

PERFORMANCE CRITERIA FOR THE LEADER/FOLLOWER ERRORS, Maximum Absolute ErRor (M.A.E.), InTEgral of AbSOLUTE ERROR (I.A.E.), INTEGRAL OF SQUARED ERROR (I.S.E.)

\begin{tabular}{ccccc}
\hline Criteria & \multicolumn{2}{c}{ Leader } & \multicolumn{2}{c}{ Follower } \\
\hline & $\mathrm{X}$ & $\mathrm{Y}$ & $\mathrm{X}$ & $\mathrm{Y}$ \\
\hline M.A.E. $[\mu \mathrm{m}]$ & 179.35 & 183.33 & 54.11 & 36.06 \\
\hline I.A.E. $[\mu \mathrm{ms}]$ & 1299.4 & 1551.9 & 295.63 & 180.64 \\
\hline I.S.E. $\left[\mu \mathrm{m}^{2} \mathrm{~s}\right]$ & $1.24 \cdot 10^{5}$ & $1.74 \cdot 10^{5}$ & 7626.2 & 1914.7 \\
\hline
\end{tabular}

TABLE III

PERFORMANCE CRITERIA FOR THE OPTIMIZATION ERRORS, MAXIMUM Absolute ErRor (M.A.E.), INTEGRAL OF SQUARED ERROR (I.S.E.)

\begin{tabular}{ccccc}
\hline Criteria & \multicolumn{2}{c}{ Leader } & \multicolumn{2}{c}{ Follower } \\
\hline & $\mathrm{X}$ & $\mathrm{Y}$ & $\mathrm{X}$ & $\mathrm{Y}$ \\
\hline M.A.E. $[\mathrm{pN}]$ & 350.46 & 206.20 & 766.79 & 316.13 \\
\hline I.S.E. $\left[\mathrm{pN}^{2} \mathrm{~s}\right]$ & $2.10 \cdot 10^{5}$ & $0.45 \cdot 10^{5}$ & $9.92 \cdot 10^{5}$ & $1.15 \cdot 10^{5}$
\end{tabular}
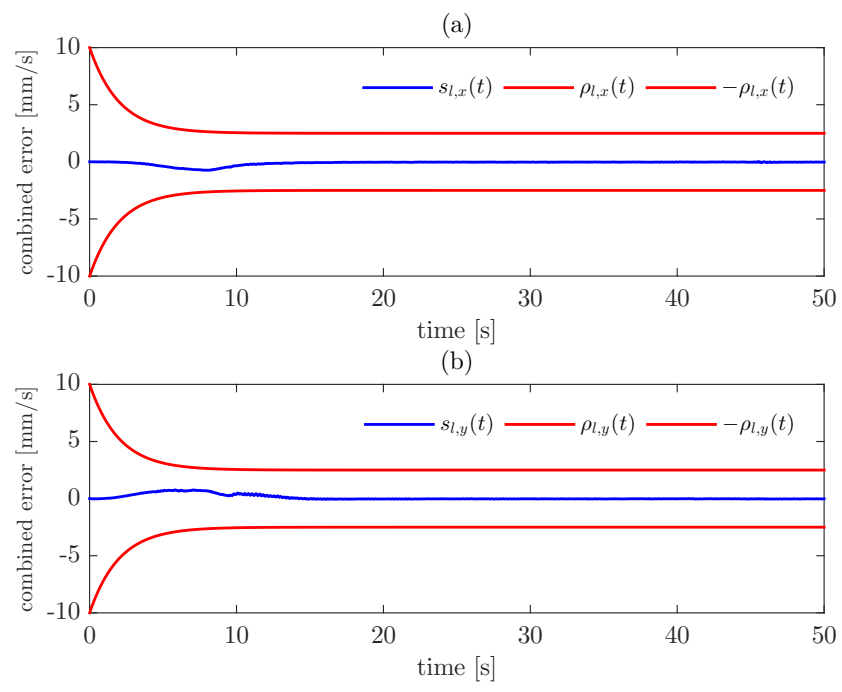

Fig. 3. Representative plots of the combined position and velocity errors (blue line) for $\mathrm{x}$ - and $\mathrm{y}$ - directions $\left(s_{l, x}(t), s_{l, y}(t)\right)$ are shown in (a) and (b), respectively. The exponentially decaying performance functions $\left(\rho_{l, x}(t)\right.$, $\left.\rho_{l, y}(t)\right)$ are also shown on each plot with red lines. 


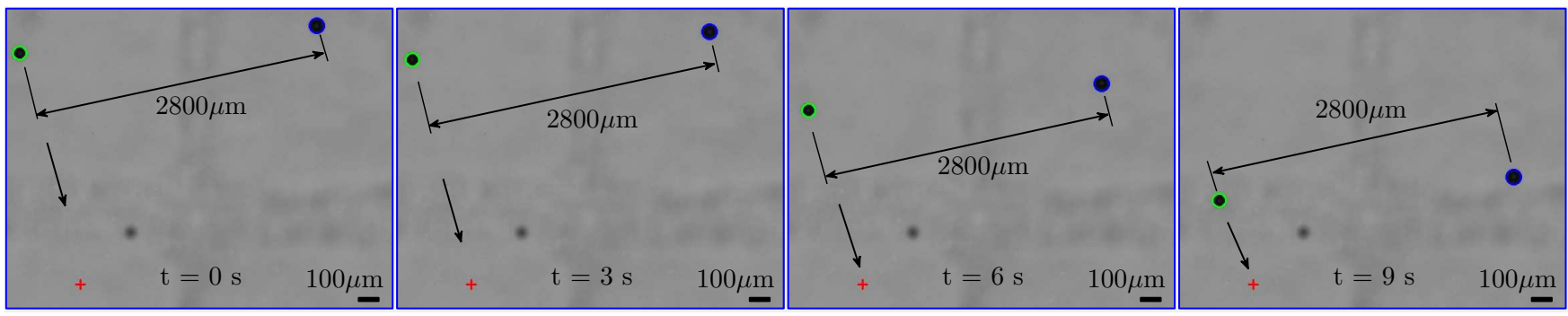

Fig. 4. Representative characteristic snapshots from leader-follower control experiments are shown. The time stamp (t) of each instant is shown at the bottom of each snapshot. The green and blue circles indicate the leader and the follower agents, respectively. Further, the red cross represents the end point of the quintic polynomial reference trajectory. The black arrows indicate the motion directions for each particle. The maximum value of the steady-state error for the leader agent is $14.45 \mu \mathrm{m}$ and $10.19 \mu \mathrm{m}$ in $\mathrm{x}$ - and $\mathrm{y}$ - directions, respectively. The maximum value of the steady-state error for the formation error is 6.47 $\mu \mathrm{m}$ and $7.77 \mu \mathrm{m}$ in $\mathrm{x}$ - and $\mathrm{y}$-directions, respectively. Please refer to the accompanying video that shows the results of the formation control experiments.

The leader-follower controller described in Section III is compared with an alternate controller. It is comprised of a proportional controller for the leader agent and prescription of zero force (i.e. $\mathbf{F}_{f}=\mathbf{0}$ ) for the follower. These control forces are converted to currents using the optimization routine described in Section III-B. The results are shown in Fig. 6 and Fig. 7, respectively. It can be observed from Fig. 6 that the error on the leader is not too large. However, the formation error given by (14) shown in Fig. 7 is large and further it seems to diverge.

\section{Independent Control Results}

We present the representative results for the independent control of two microparticles in a letter writing task. One of the particles moves along a $U$-shaped path and the other one moves along a $T$-shaped path on the surface of the water. We used the prescribed performance controller (11) for both of the agents. Thus, the control forces are selected as follows:

$$
\mathbf{F}_{j, i}=-k_{j, i} \ln \left(\frac{1+\frac{s_{j, i}}{\rho_{j, i}}}{1-\frac{s_{j, i}}{\rho_{j, i}}}\right) \quad \text { for } \quad i \in\{x, y\}
$$

where $j \in\{1,2\}$ is the index of the corresponding agent.
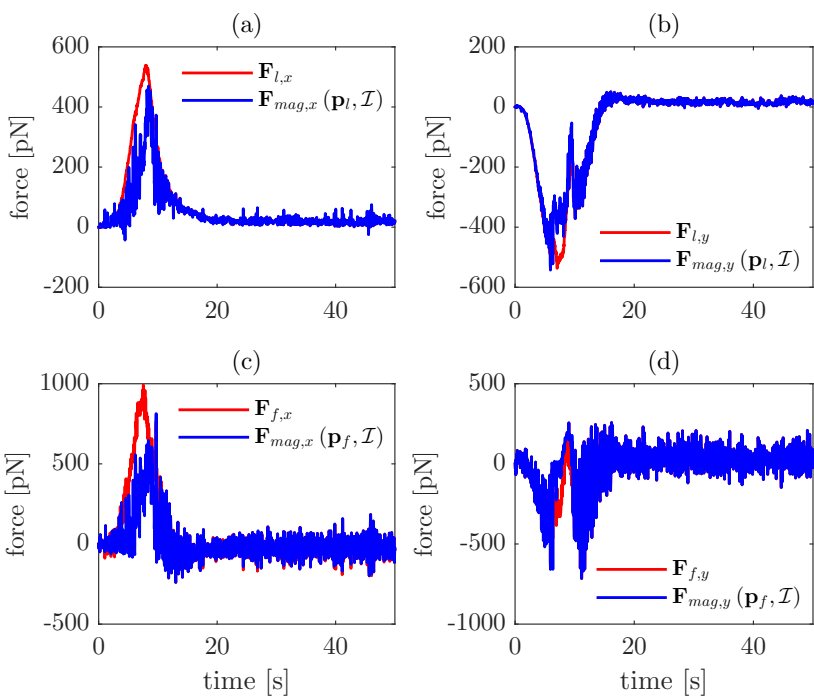

Fig. 5. Representative plots of the controller forces (red line) for the leader agent given by $\left(\mathbf{F}_{l, x}, \mathbf{F}_{l, y}\right)$ and the forces (blue line) obtained with $\left(\mathbf{F}_{\text {mag }, x}\left(\mathbf{p}_{l}, \mathcal{I}^{*}\right), \mathbf{F}_{\text {mag }, y}\left(\mathbf{p}_{l}, \mathcal{I}^{*}\right)\right)$ using the optimal current $\left(\mathcal{I}^{*}\right)$ for $\mathrm{x}$ and y- directions are shown in (a) and (b), respectively. The controller forces (red line) for the follower agent given by $\left(\mathbf{F}_{f, x}, \mathbf{F}_{f, y}\right)$ and the forces (blue line) obtained with $\left(\mathbf{F}_{m a g, x}\left(\mathbf{p}_{f}, \mathcal{I}^{*}\right), \mathbf{F}_{m a g, y}\left(\mathbf{p}_{f}, \mathcal{I}^{*}\right)\right)$ using the optimal current $\left(\mathcal{I}^{*}\right)$ for $\mathrm{x}$ - and $\mathrm{y}$ - directions are shown in the bottom plots in (c) and (d), respectively.
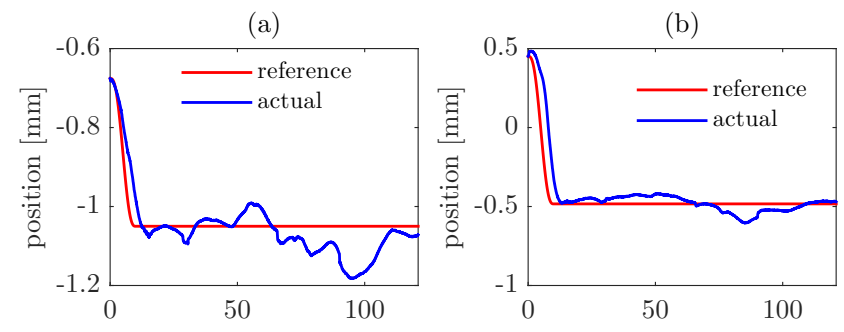

(c)

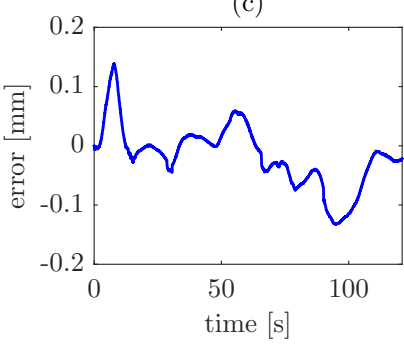

(d)

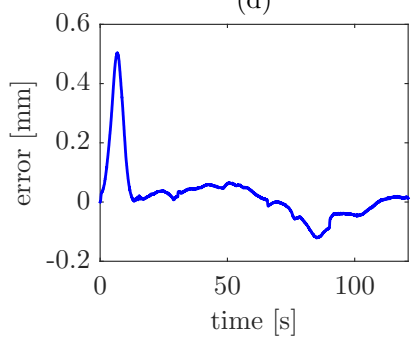

Fig. 6. Representative plots of the leader reference (red) and actual (blue) positions for $\mathrm{x}$ - and $\mathrm{y}$ - directions are shown in (a) and (b), respectively. The error between them are shown on the bottom plots for $\mathrm{x}$ - and $\mathrm{y}$ - directions in (c) and (d), respectively.
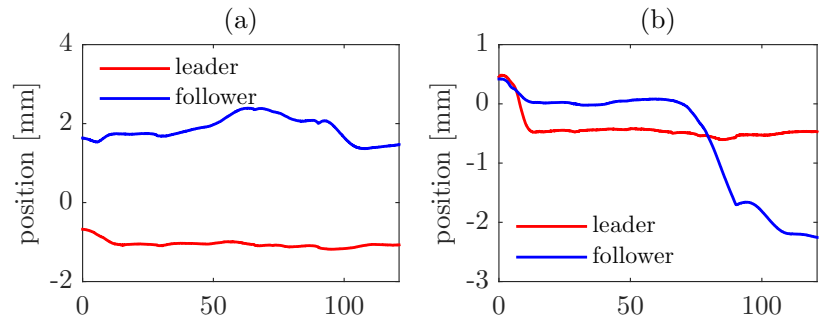

(c)

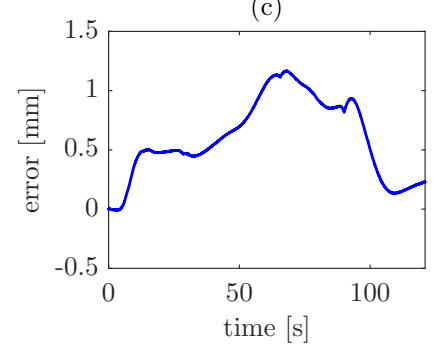

(d)

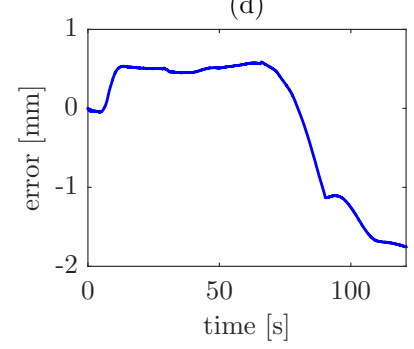

Fig. 7. Representative plots of the leader (red) and follower (blue) positions for $\mathrm{x}$ - and $\mathrm{y}$ - directions are shown in (a) and (b), respectively. The formation error between them are shown on the bottom plots for $\mathrm{x}$ - and $\mathrm{y}$ - directions in (c) and (d), respectively.

Each agent receives a separate reference trajectory determined by four waypoints to form the letters. These waypoints are 


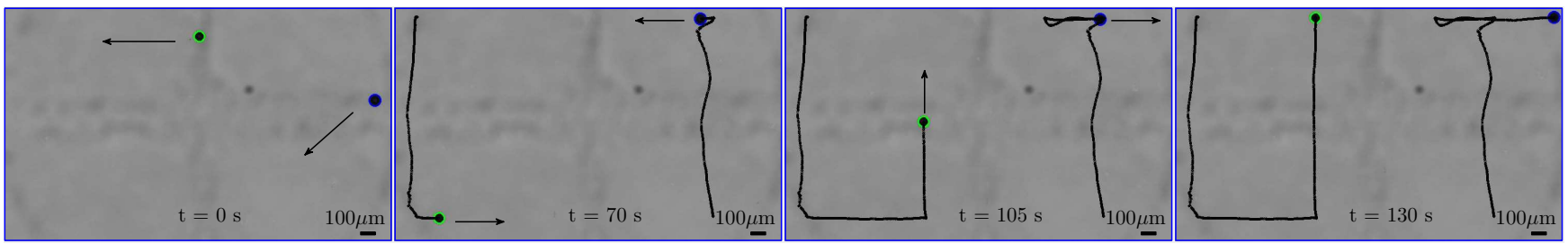

Fig. 8. Representative characteristic snapshots from the independent motion control experiments are shown. The time stamp (t) of each instant is shown at the bottom of each snapshot. The green and blue circles indicate the two different agents, respectively. Further, the trace of the agents are shown with black lines. The black arrows indicate the motion directions for each particle. The particle with the green circle writes the letter $\mathrm{U}$ and the particle with the blue circle writes the letter T. Please refer to the accompanying video that shows the results of the independent control experiments.

(a)

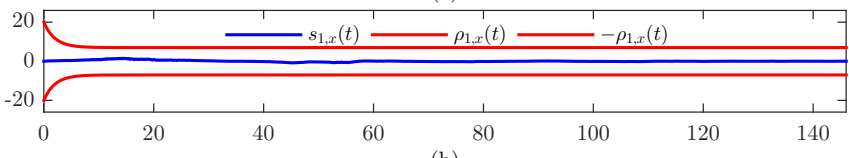
(b)

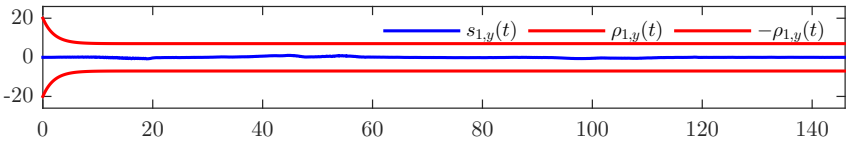

(c)

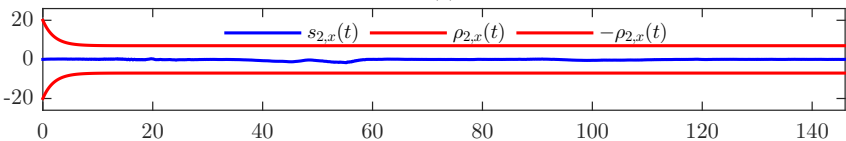

(d)

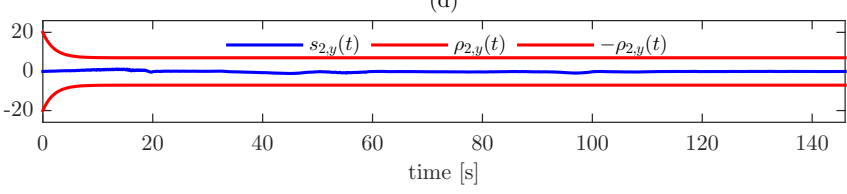

Fig. 9. Representative plots of the combined position and velocity error (blue lines) for the first particle in $\mathrm{x}$ - and $\mathrm{y}$ - directions $\left(s_{1, x}(t), s_{1, y}(t)\right)$ are shown in (a) and (b) and for the second particle $\left(s_{2, x}(t), s_{2, y}(t)\right)$ in (c) and (d), respectively. The exponentially decaying performance functions $\left(\rho_{1, x}(t), \rho_{1, y}(t), \rho_{2, x}(t), \rho_{2, y}(t)\right)$ are shown on each plot with red lines.

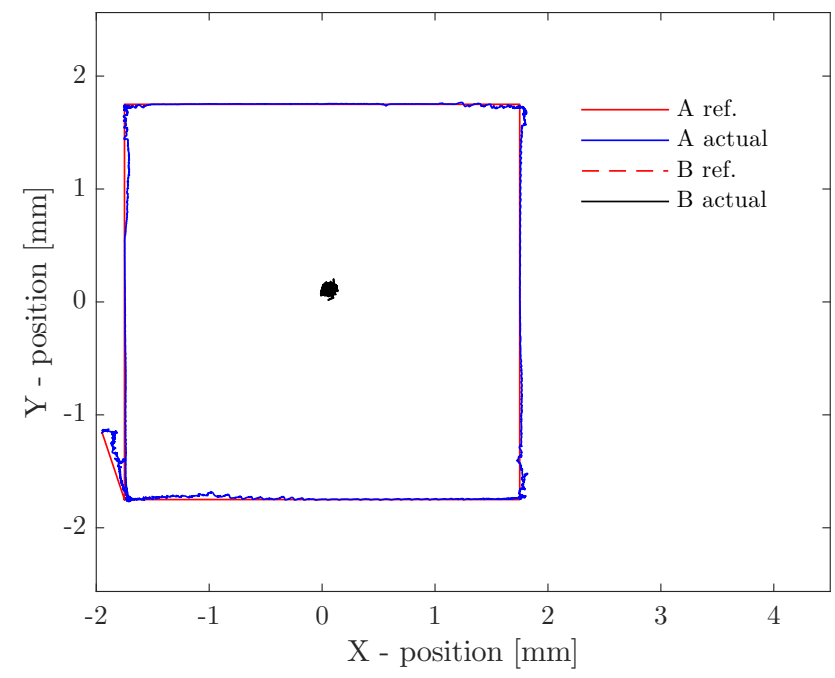

Fig. 10. Representative plots of the reference (red line) and actual (blue line) positions of Particle A and reference (red dashed line) and actual (black line) for Particle B in the xy-plane.

connected to each other using quintic polynomial trajectories with zero initial and final velocities and accelerations. The relation between the magnetic control forces and the currents are solved using the optimization algorithm described in

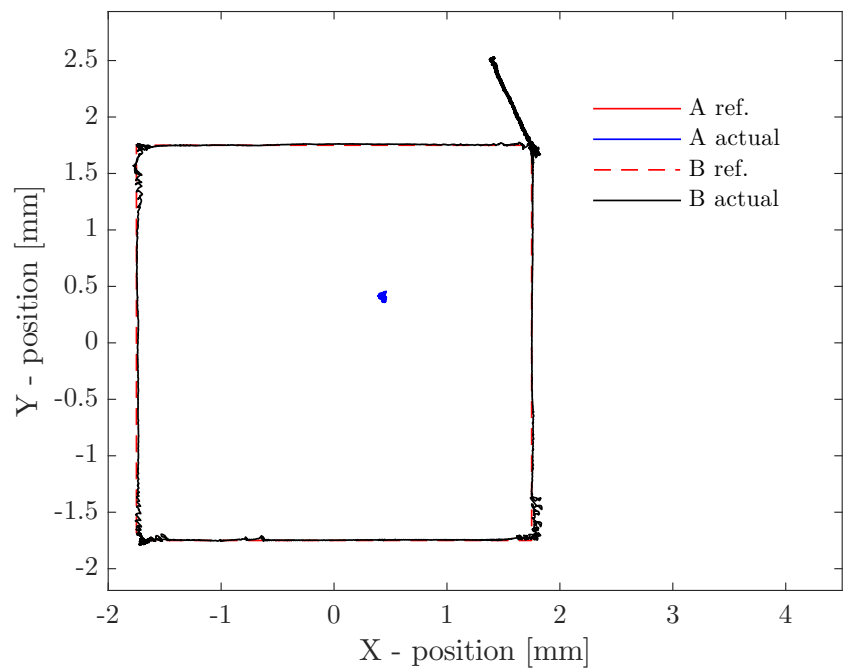

Fig. 11. Representative plots of the reference (red line) and actual (blue line) positions of Particle A and reference (red dashed line) and actual (black line) for Particle B in the xy-plane.

Section III-B. The final time of each segment of the reference trajectory is selected as $t_{f}=30$ seconds. Video snapshots of the two agents during the independent control experiment are shown in Fig. 8. Further, the combined position and velocity errors together with the prescribed performance bounds for both particles in $\mathrm{x}$ - and $\mathrm{y}$ - directions are shown in Fig. 9. Besides the letter-shaped path tracking results, we performed three benchmark tests. In the first test, particle A moves in clockwise direction along a square path while particle B remains still and the results are shown in Fig. 10. In the second test, particle B moves in clockwise direction along a square path while particle A remains still and the results are shown in Fig. 11. In the third test, particle A moves in clockwise direction along a square path while particle B moves in counterclockwise direction along another square path and the results are shown in Fig. 12. The quantitative evaluation of the performance for these three benchmark tests is shown in Table IV.

\section{COnClusions}

We investigated the design of a formation controller using the leader-follower approach for two micro-sized agents. A prescribed performance controller is used to control the position of the leader micro-agent. The trajectory of the leader is used as a reference for the follower micro-agent. The control currents are computed using the relaxed semi-definite programming approach. 


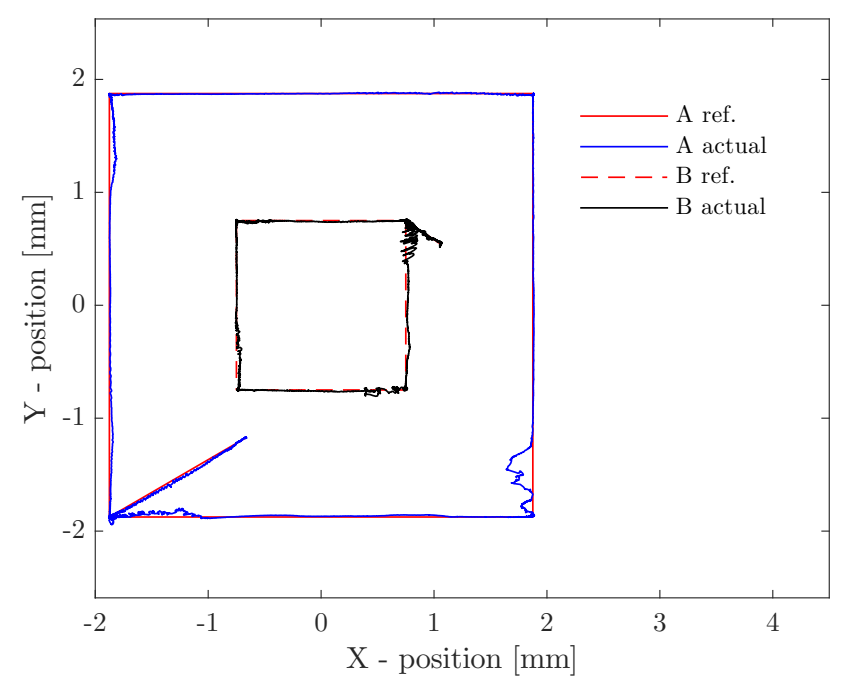

Fig. 12. Representative plots of the reference (red line) and actual (blue line) positions of Particle A and reference (red dashed line) and actual (black line) for Particle B in the xy- plane.

TABLE IV

PERFORMANCE CRITERIA FOR THE BENCHMARK TESTS, MAXIMUM Absolute Error (M.A.E.), InTEgral of Absolute ERror (I.A.E.),

\begin{tabular}{ccccc}
\multicolumn{5}{c}{ INTEGRAL OF SQUARED ERROR (I.S.E.) } \\
\hline Criteria & \multicolumn{2}{c}{ Particle A } & \multicolumn{2}{c}{ Particle B } \\
\hline Test 1 & $\mathrm{X}$ & $\mathrm{Y}$ & $\mathrm{X}$ & $\mathrm{Y}$ \\
\hline M.A.E. $[\mu \mathrm{m}]$ & 131.84 & 74.27 & 131.3 & 96.68 \\
\hline I.A.E. $[\mu \mathrm{ms}]$ & 8262.1 & 3895.8 & 7200.3 & 3219.3 \\
\hline I.S.E. $\left[\mu \mathrm{m}^{2} \mathrm{~s}\right]$ & $3.62 \cdot 10^{5}$ & 81740 & $2.92 \cdot 10^{5}$ & 63772 \\
\hline Test 2 & $\mathrm{X}$ & $\mathrm{Y}$ & $\mathrm{X}$ & $\mathrm{Y}$ \\
\hline M.A.E. $[\mu \mathrm{m}]$ & 65.97 & 61.46 & 103.02 & 110.01 \\
\hline I.A.E. $[\mu \mathrm{ms}]$ & 6080 & 3485.8 & 7033.8 & 6129.6 \\
\hline I.S.E. $\left[\mu \mathrm{m}^{2} \mathrm{~s}\right]$ & $1.57 \cdot 10^{5}$ & 73885 & $2.33 \cdot 10^{5}$ & $2.1 \cdot 10^{5}$ \\
\hline Test 3 & $\mathrm{X}$ & $\mathrm{Y}$ & $\mathrm{X}$ & $\mathrm{Y}$ \\
\hline M.A.E. $[\mu \mathrm{m}]$ & 236.11 & 77.38 & 195.41 & 67.75 \\
\hline I.A.E. $[\mu \mathrm{ms}]$ & 9624.3 & 3431.1 & 5994.3 & 3467.8 \\
\hline I.S.E. $\left[\mu \mathrm{m}^{2} \mathrm{~s}\right]$ & $5.98 \cdot 10^{5}$ & 65852 & $2.44 \cdot 10^{5}$ & 58282
\end{tabular}

Experiments on two spherical magnetic microparticles with an average diameter of $100 \mu \mathrm{m}$ are performed. The maximum value of the steady-state errors obtained in the formation control experiments are $14.45 \mu \mathrm{m}$ and $10.19 \mu \mathrm{m}$ in $\mathrm{x}$ - and $\mathrm{y}$ - directions for the leader agent and $6.47 \mu \mathrm{m}$ and $7.77 \mu \mathrm{m}$ in $\mathrm{x}$ - and $\mathrm{y}$ - directions for the formation errors, respectively.

In future work, we will apply this control method to a larger number and different type of micro-sized agents in the 3D case. The robustness of the control algorithm to environmental disturbances such as fluid flow will also be evaluated. Furthermore, the performance of the control algorithm will also be tested using different imaging modalities.

\section{ACKNOWLEDGMENT}

The authors acknowledge the helpful discussions with Dr. Peter J.C. Dickinson about the semi-definite programming problem.

\section{REFERENCES}

[1] A. Becker, C. Onyuksel, T. Bretl, and J. McLurkin, "Controlling many differential-drive robots with uniform control inputs," The International Journal of Robotics Research, vol. 33, no. 13, pp. 1626-1644, 2014.

[2] S. Shahrokhi and A. T. Becker, "Stochastic swarm control with global inputs," in the Proceedings of the IEEE/RSJ International Conference on Intelligent Robots and Systems (IROS), Sept 2015, pp. 421-427.
[3] D. Wong, E. B. Steager, and V. Kumar, "Independent control of identical magnetic robots in a plane," IEEE Robotics and Automation Letters, vol. 1, no. 1, pp. 554-561, Jan 2016

[4] E. Diller, J. Giltinan, and M. Sitti, "Independent control of multiple magnetic microrobots in three dimensions," The International Journal of Robotics Research, vol. 32, no. 5, pp. 614-631, 2013.

[5] N. A. Torres and D. O. Popa, "Cooperative control of multiple untethered magnetic microrobots using a single magnetic field source," in the Proceedings of the IEEE International Conference on Automation Science and Engineering (CASE), Aug 2015, pp. 1608-1613.

[6] Q. Chao, J. Yu, C. Dai, T. Xu, L. Zhang, C. C. L. Wang, and X. Jin, "Steering micro-robotic swarm by dynamic actuating fields," in the Proceedings of the IEEE International Conference on Robotics and Automation (ICRA), May 2016, pp. 5230-5235.

[7] L. Mellal, D. Folio, K. Belharet, and A. Ferreira, "Optimal control of multiple magnetic microbeads navigating in microfluidic channels," in the Proceedings of the IEEE International Conference on Robotics and Automation (ICRA), May 2016, pp. 1921-1926.

[8] S. Martel and M. Mohammadi, "A robotic micro-assembly process inspired by the construction of the ancient pyramids and relying on several thousand flagellated bacteria acting as micro-workers," in the Proceedings of the IEEE/RSJ International Conference on Intelligent Robots and Systems (IROS), Oct 2009, pp. 426-427.

[9] A. Becker, Y. Ou, P. Kim, M. J. Kim, and A. Julius, "Feedback control of many magnetized: Tetrahymena pyriformis cells by exploiting phase inhomogeneity," in the Proceedings of the IEEE/RSJ International Conference on Intelligent Robots and Systems (IROS), Nov 2013, pp. 3317-3323.

[10] P. S. S. Kim, A. Becker, Y. Ou, A. A. Julius, and M. J. Kim, "Swarm control of cell-based microrobots using a single global magnetic field," in the Proceedings of the 10th International Conference on Ubiquitous Robots and Ambient Intelligence (URAI), Oct 2013, pp. 21-26.

[11] S. Chowdhury, W. Jing, and D. J. Cappelleri, "Controlling multiple microrobots: recent progress and future challenges," Journal of MicroBio Robotics, vol. 10, no. 1, pp. 1-11, 2015.

[12] H. Nijmeijer and A. Rodriguez-Angeles, External synchronization of rigid joint robots. World Scientific, 2012, ch. 3, pp. 35-60.

[13] B. R. Munson, T. H. Okiishi, W. W. Huebsch, and A. P. Rothmayer, "Chapter 9 - flow over immersed bodies," in Fundamentals of fluid mechanics. John Wiley \& Sons, Inc., 2013, pp. 480 - 554.

[14] M. Dkhil, M. Kharboutly, A. Bolopion, S. Régnier, and M. Gauthier, "Closed-loop control of a magnetic particle at the air-liquid interface," IEEE Transactions on Automation Science and Engineering, vol. PP, no. 99, pp. 1-13, 2017.

[15] M. Kummer, J. Abbott, B. Kratochvil, R. Borer, A. Sengul, and B. Nelson, "Octomag: An electromagnetic system for 5-dof wireless micromanipulation," IEEE Transactions on Robotics, vol. 26, no. 6, pp. 1006-1017, 2010.

[16] J. Keuning, J. de Vries, L. Abelmann, and S. Misra, "Image-based magnetic control of paramagnetic microparticles in water," in the Proceedings of the IEEE/RSJ International Conference on Intelligent Robots and Systems (IROS), September 2011, pp. 421-426.

[17] A. Sanchez, V. Magdanz, O. G. Schmidt, and S. Misra, "Magnetic control of self-propelled microjets under ultrasound image guidance," in the Proceedings of the $5^{\text {th }}$ IEEE RAS International Conference on Biomedical Robotics and Biomechatronics (EMBS), August 2014, pp. 169-174.

[18] C. P. Bechlioulis and G. A. Rovithakis, "A low-complexity global approximation-free control scheme with prescribed performance for unknown pure feedback systems," Automatica, vol. 50, no. 4, pp. 1217 $-1226,2014$.

[19] A. Denasi and S. Misra, "A robust controller for micro-sized agents: The prescribed performance approach," in the Proceedings of the Annual International Conference on Manipulation, Automation and Robotics at Small Scales (MARSS), July 2016, pp. 1-6.

[20] C. Bechlioulis, M. Liarokapis, and K. Kyriakopoulos, "Robust model free control of robotic manipulators with prescribed transient and steady state performance," in the Proceedings of the IEEE/RSJ International Conference on Intelligent Robots and Systems (IROS), September 2014, pp. 41-46.

[21] P. Y. Papalambros and D. J. Wilde, "Optimization models," in Principles of Optimal Design: Modeling and Computation. Cambridge: Cambridge University Press, 007 2000, pp. 1-43.

[22] Z. Q. Luo, W. K. Ma, A. M. C. So, Y. Ye, and S. Zhang, "Semidefinite relaxation of quadratic optimization problems," IEEE Signal Processing Magazine, vol. 27, no. 3, pp. 20-34, May 2010 